\title{
Visualizing the Coupling between Red and Blue Stark States Using Photoionization Microscopy
}

\author{
A. S. Stodolna, ${ }^{1}$ F. Lépine, ${ }^{2}$ T. Bergeman, ${ }^{3}$ F. Robicheaux,${ }^{4}$ A. Gijsbertsen, ${ }^{1}$ J. H. Jungmann, ${ }^{1}$ \\ C. Bordas, ${ }^{2}$ and M. J. J. Vrakking ${ }^{1,5, *}$ \\ ${ }^{1}$ FOM Institute AMOLF, Science Park 104, 1098 XG Amsterdam, The Netherlands \\ ${ }^{2}$ Institut Lumiére Matiére, Université Lyon 1, CNRS, UMR 5306, 10 rue Ada Byron, 69622 Villeurbanne Cedex, France \\ ${ }^{3}$ Physics and Astronomy, Stony Brook University, Stony Brook, New York 11794, USA \\ ${ }^{4}$ Department of Physics, Purdue University, West Lafayette, Indiana 47907, USA \\ ${ }^{5}$ Max-Born-Institut, Max Born Straße 2A, D-12489 Berlin, Germany
}

(Received 24 October 2013; published 4 September 2014)

\begin{abstract}
In nonhydrogenic atoms in a dc electric field, the finite size of the ionic core introduces a coupling between quasibound Stark states that leads to avoided crossings between states that would otherwise cross. Near an avoided crossing, the interacting states may have decay amplitudes that cancel each other, decoupling one of the states from the ionization continuum. This well-known interference narrowing effect, observed as a strongly electric field-dependent decrease in the ionization rate, was previously observed in several atoms. Here we use photoionization microscopy to visualize interference narrowing in helium atoms, thereby explicitly revealing the mechanism by which Stark states decay. The interference narrowing allows measurements of the nodal patterns of red Stark states, which are otherwise not observable due to their intrinsic short lifetime.
\end{abstract}

DOI: 10.1103/PhysRevLett.113.103002

PACS numbers: $32.80 . \mathrm{Fb}, 32.60 .+\mathrm{i}$

Throughout the last decades, the dynamics of Rydberg states has been a topic of great interest. Studies have explored the connection between quantum and classical mechanics, have revealed regimes where excitation and ionization are best described in terms of a photon or field picture, have allowed some of the first time-resolved observations of wave packet dynamics, and have led to novel concepts relevant to coherent control and quantum computation [1-5]. The behavior of Rydberg states exposed to external (magnetic or electric) fields has been of particular interest.

Here, our focus will be on dc electric fields, which lead to a coupling between Rydberg states where the orbital angular momentum $l$ is no longer a good quantum number. In atomic hydrogen, for a given value of the principal quantum number $n$, the Rydberg states form Stark manifolds characterized by parabolic quantum numbers $n_{1}$ and $n_{2}$ (where $n=n_{1}+n_{2}+|m|+1$, with $m$ the magnetic quantum number). The Stark energy is, to first order in the electric field $F$, given by $E=-\left(1 / 2 n^{2}\right)+\frac{3}{2} F n\left(n_{1}-n_{2}\right)$ (atomic units are used unless indicated otherwise). Red Stark states $\left(n_{1}<n_{2}\right)$ are localized downhill on the Coulomb $+\mathrm{dc}$ field potential and display higher ionization rates than blue Stark states $\left(n_{1}>n_{2}\right)$, located uphill. For $F>1 / 3 n^{5}$ adjacent Stark manifolds overlap, and blue and red Stark states cross each other without coupling.

In nonhydrogenic atoms, the interaction of the Rydberg electron with the core is no longer purely Coulombic. Without electric field, the energy levels are now given by a Rydberg-like formula, where $n$ is replaced by $n^{*}=n-\mu$, with $\mu$ the quantum defect [6]. In the presence of an electric field, Stark states ionize by a process similar to autoionization [7]. This significantly decreases the lifetimes compared to the hydrogenic case, where the ionization of Stark states occurs exclusively via tunneling (leaving the value of $n_{1}$ unchanged). The nonzero quantum defects lead to couplings among the Stark states and avoided crossings arise when two Stark states cross [8].

Studies of Stark states around an avoided crossing have included observations of interference narrowing [9-11], a localized, strongly electric field-dependent increase in the lifetime of the short-lived red Stark states. As explained in [10], this occurs because the matrix element describing the ionization rate can be written as the sum of individual contributions from uncoupled Stark states which may to a considerable extent cancel each other. Here, we apply the recently developed photoionization microscopy technique [12-14] to observe changes in the Stark wave function when interference narrowing occurs. Photoionization microscopy has its origin in predictions made by Demkov and co-workers about 30 years ago [15-17]. They argued that imaging the projection of very slow (typically $\mathrm{meV}$ ) photoelectrons, formed by photoionization or negative ion photodetachment in the presence of a dc electric field, would lead to the observation of interference patterns, resulting either from the nodal structure of prepared Stark states (in the case of photoionization), or from quantum-mechanical path length differences along multiple interfering pathways connecting the atom to a particular position on the detector (in both cases). This 
concept was first validated in photodetachment experiments by Blondel and co-workers [18]. Photoionization microscopy was first realized by Nicole and co-workers [12], who observed interference patterns resulting from continuum path length differences in xenon [19]. The possibility to observe the nodal structure of quasibound Stark states was recently validated in experiments on hydrogen [13,20] and lithium [14]. Here, we exploit this capability to directly visualize changes in the Stark wave function when interference narrowing occurs.

Photoionization microscopy experiments are best described using parabolic coordinates $\eta$ and $\xi(\eta=r-z$ and $\xi=r+z$, with $r$ the distance from the electron to the ionic core, and $z$ the displacement along the electric field axis; $y$ and $x$ are two Cartesian coordinates perpendicular to $z)$. Consequently, in hydrogen, the Stark wave function can be written as $\Psi(\xi, \eta, \varphi)=(2 \pi \eta \xi)^{-1 / 2} \chi_{1}(\xi) \chi_{2}(\eta) \mathrm{e}^{i m \varphi}$, where $\varphi=\tan ^{-1}(y / x)$ is the azimuthal angle. The parabolic quantum numbers $n_{1}$ and $n_{2}$ are the number of nodes in $\chi_{1}(\xi)$ and $\chi_{2}(\eta)$.

The nature of the ionization process leaves its signature in the interference patterns that can be recorded in photoionization microscopy experiments. In hydrogen Stark states, ionization involves tunneling through the effective potential along the $\eta$ coordinate, $V(\eta)=-\left(Z_{2} / 2 \eta\right)+\left(\left(m^{2}-\right.\right.$ 1) $\left./ 8 \eta^{2}\right)-(F \eta / 8)$ (see, e.g., Fig. 1(b) in [13]), where $Z_{2}$ is a separation constant [21]. This tunneling only affects $\chi_{2}(\eta)$, and therefore experimentally observed dark fringes directly reveal the nodal structure of $\chi_{1}(\xi)$, as observed in $[13,14]$. If the ionization takes place by direct excitation into the continuum (i.e. excitation above the barrier in $V(\eta)$ ), interferences due to semiclassical path length differences are observed [19]. A special case arises in nonhydrogenic systems, where the Schrödinger equation is no longer separable in parabolic coordinates, and where ionization from a quasibound Stark state that is bound in the $V(\eta)$ potential can occur by core scattering into states above the $V(\eta)$ barrier. The latter process, is the dominant ionization mechanism in nonhydrogenic atoms and leads to interferences that-like the case of direct excitation above the $V(\eta)$ barrier-are governed by semiclassical path length differences.

We consider the case of a singlet state in helium, where $\mu_{s}=0.14, \mu_{p}=-0.012, \mu_{d}=0.002$ and $\mu_{l \geq 3} \approx 0$ [22]. Metastable $\mathrm{He}^{*}\left(2^{1} \mathrm{~S}\right)$ was generated in a water-cooled $\mathrm{cw}$ discharge source (see Fig. 1) [23]. The $\mathrm{He}^{*}$ intensity was $7.5 \times 10^{12}$ atoms/ sec/steradian. The $\mathrm{He}^{*}$ atoms were photoexcited using the frequency-doubled output of a cw ring dye laser (Coherent 899-29). Resulting photoelectrons were detected using a velocity map imaging (VMI) spectrometer [24], with an Einzel lens placed half-way towards the two-dimensional detector, which enabled a tenfold magnification of the observed images [25]. The detector consisted of two 4-cm microchannel plates (MCPs), followed by a phosphor screen and a CCD camera. To suppress Penning ionization of background gas, the

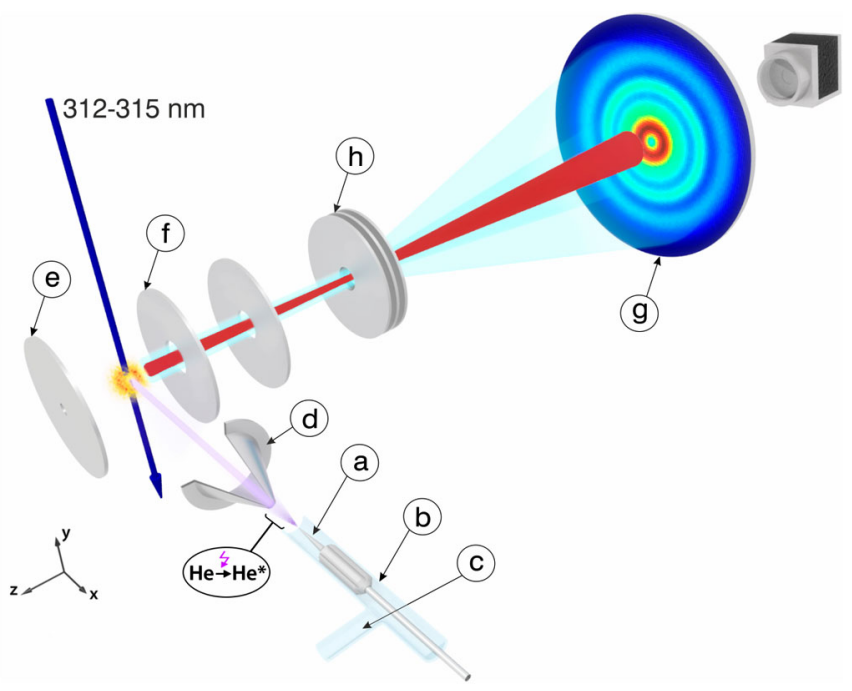

FIG. 1 (color online). Schematic representation of the experimental setup. Metastable $2^{1} \mathrm{~S}$ helium atoms are created by means of a dc electric discharge maintained between a Tantalum needle (a), surrounded by a quartz tube (b) and filled with $\mathrm{He}$ gas provided by a gas inlet (c), and a grounded skimmer (d). The ignition starts at $-2 \mathrm{kV}$ and is sustained at $-0.7 \mathrm{kV}$ and $20 \mathrm{~mA}$. The $\mathrm{He}^{*}$ beam, formed by the skimmer $(\phi=1 \mathrm{~mm})$, crosses a tunable UV laser inside a velocity map imaging (VMI) spectrometer, permitting the excitation of quasibound Stark states that subsequently ionize. By applying a voltage difference across the repeller (e) and extractor (f) plates the photoelectrons are accelerated towards a position-sensitive detector $(\mathrm{g})$ comprising 4-cm diameter MCP plates, a phosphor screen and a CCD camera. Halfway towards the detector, the photoelectrons pass through an Einzel lens (h), enabling magnification of the recorded photoelectron images by one order of magnitude.

VMI was surrounded by a liquid nitrogen cold trap, and images were recorded as the difference between acquisitions with and without the UV laser. The dc field strength was maintained at approximately $468 \mathrm{~V} / \mathrm{cm}$. The laser polarization was along the dc electric field, resulting in the population of $m=0$ Stark states. Images were recorded following excitation of quasibound Stark states lying between the saddle point in the Coulomb $+\mathrm{dc}$ field potential at an energy $E_{s p}=-2 \sqrt{F}=-132.4 \mathrm{~cm}^{-1}$ and the field-free ionization limit $(E=0)$. The signal levels did not permit measurements away from Stark resonances.

In Figs. 2(a)-2(d), a series of representative images reveal the appearance of interferences, with a number of nodes that increases with the photoelectron kinetic energy (defined with respect to the saddle point). As illustrated in Fig. 2(e), the energy dependence of this interference structure can be understood semiclassically by considering the phase accumulation along different trajectories that reach the same position on the detector [19]. These results, which account for about $95 \%$ of the images, are fully consistent with the results previously reported for xenon [12], and-consequently-will not be discussed further here. 

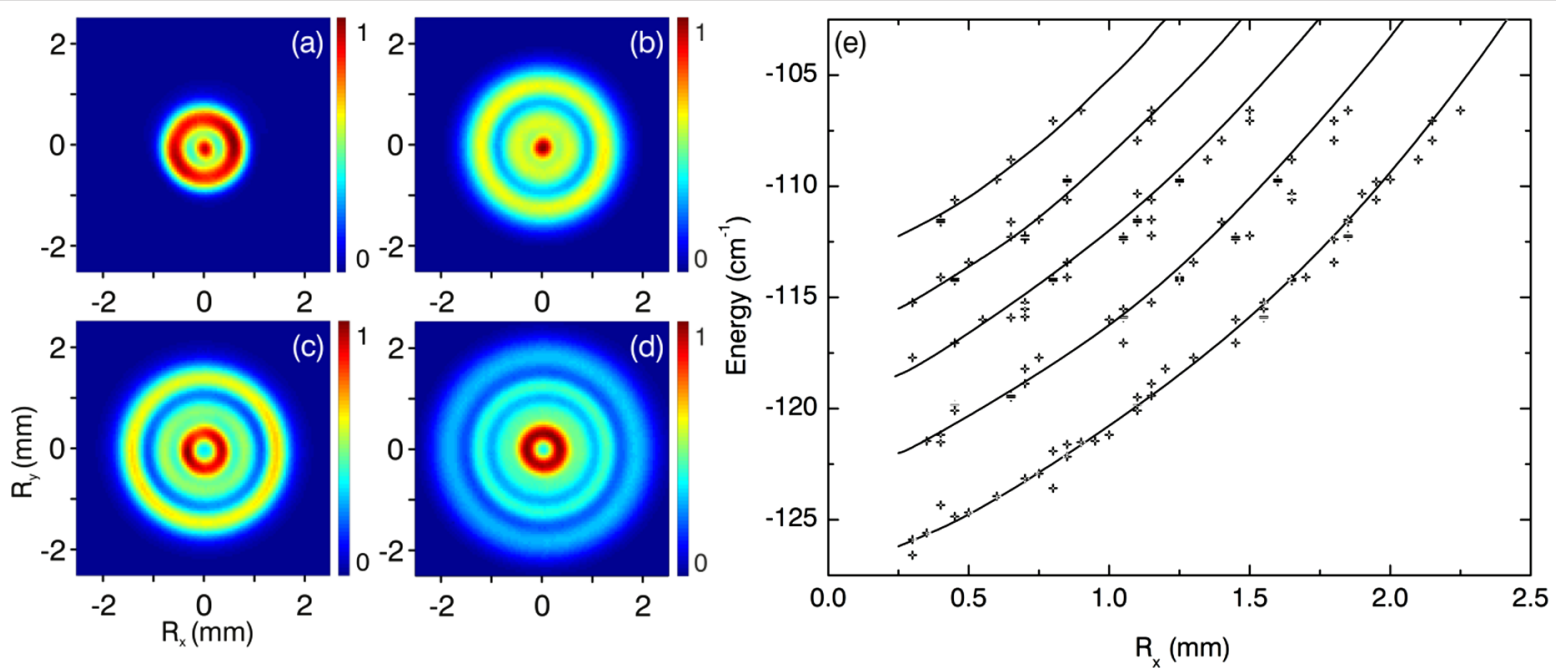

FIG. 2 (color online). Photoelectron images recorded for Stark states that decay via core-induced coupling to fast-ionizing red states. The examples shown in (a)-(d) are the $(28,22,5,0),(28,25,2,0),(29,21,7,0)$ and $(21,23,5,0)$ states, acquired at energies of $-126.59 \mathrm{~cm}^{-1},-121.46 \mathrm{~cm}^{-1},-119.42 \mathrm{~cm}^{-1}$ and $-115.87 \mathrm{~cm}^{-1}$, respectively, in the presence of a $468 \mathrm{~V} / \mathrm{cm} \mathrm{dc} \mathrm{electric} \mathrm{field.} \mathrm{In} \mathrm{all}$ images, the intensities are normalized to the maximum occurring intensity. The main observation is that the number of interference minima increases smoothly as a function of energy. The interferences can be explained by considering the phase difference between different trajectories by which electrons can move from the ion to the detector. This is further illustrated in (e), where the positions of experimentally observed interference minima $(+)$ are compared with semiclassically calculated positions (solid lines, using the method described in [19]).

While preparing Fig. 2(e), seven images were excluded, where the observed nodal pattern differed markedly from the semiclassical predictions. These images were recorded in the vicinity of avoided crossings between red and blue Stark states, and reveal the nodal structure along the $\xi$ coordinate of the red Stark states involved. To illustrate this, Figs. 3(a)-3(f) show raw images and extracted radial distributions for three measurements performed at energies just above the saddle point, involving the $\left(n, n_{1}, n_{2}, m\right)=(31,7,23,0),(34,1,32,0)$ and $(27,26$, $0,0)$ states. The $(34,1,32,0)$ state is an extreme red Stark state, whereas the other two states lie in the middle and on the blue side of the Stark manifold. Remarkably, the radial distribution of the red $(34,1,32,0)$ state extends further than that of the two adjacent states, and has a minimum. Both observations point towards hydrogenic behavior [13]. The observed minimum reflects the value of $n_{1}$, and describes a node in $\chi_{1}(\xi)$. Furthermore, the increased radial extension reflects a hydrogenic tunneling process, which allows the electron to reach positions on the detector that are not accessible when the electron ionizes by passing over the saddle point in $V(\eta)[26,27]$.

This interpretation is confirmed by numerically solving of the time-dependent Schrödinger equation (TDSE) [28]. In these calculations, the $\mathrm{He}^{*}$ atoms were ionized using a $1.5 \mathrm{~ns}$ long bandwidth-limited laser pulse, and the propagation was continued for $3 \mathrm{~ns}$. To extract a two-dimensional image, the flux through a screen placed at a distance of $0.8 \mu \mathrm{m}$ was evaluated. The radial distributions no longer change qualitatively beyond this distance. The numerical radial distributions (scaled to the size of the experimentally measured distributions) reproduce the essential features, i.e., the minimum in the radial distribution for the $(34,1$, $32,0)$ state and its larger radial extension.

Photoabsorption cross sections were computed by Fourier transforming the auto-correlation $\langle\psi(t=0) \mid \psi(t)\rangle$ [29]. Stark maps were obtained by reading off local maxima in the computed absorption strength as a function of laser wavelength. The Stark map shown in Fig. $3(\mathrm{~g})$ reveals that the measurement for the $(34,1,32,0)$ state was performed in the vicinity of an avoided crossing with the $(27,26,0,0)$ state. The approximate hydrogenic assignments of the diabatic, uncoupled Stark states indicated in Fig. 3(g) were obtained by exploiting the similarities between the calculated Stark map and a hydrogenic calculation (using formulas given in Ref. [30]). Monitoring the line widths of the Stark states in the photoabsorption spectra (by fitting a Lorentzian profile to the observed resonance) it was discovered that the condition under which the experiment for the $(34,1,32,0)$ state was performed coincided with a minimum in the line width of this state [Fig. 3(h)]. In other words, the observation of the nodal pattern of the $(34,1,32$, 0) state was made possible by a lifetime enhancement (i.e. interference narrowing) of this state, due to coupling with the blue $(27,26,0,0)$ state. A very similar reasoning underlies the measurement of six other photoelectron images (one of which is shown in Fig. 4), where the experiments disagree with semiclassical predictions. In each case, an avoided crossing can be identified, and the nodal structure observed on the detector was that of the red Stark state involved. Furthermore, all these states showed a strongly field-dependent increase in their lifetimes. In the 

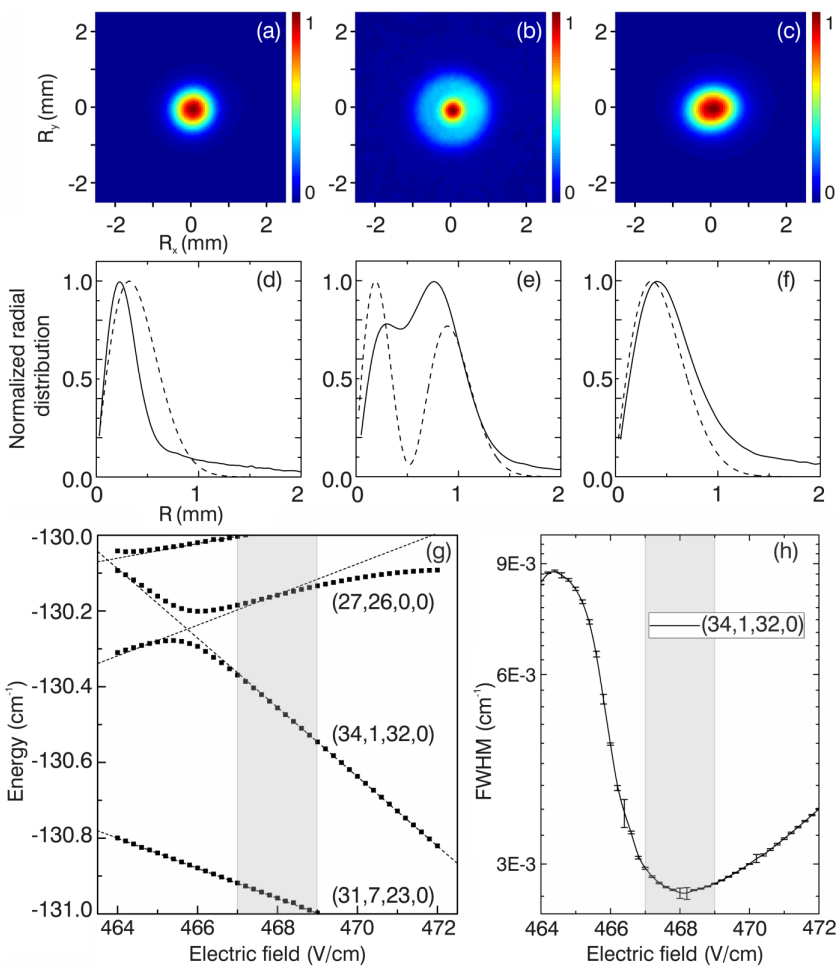

FIG. 3 (color online). (a)-(c) Three electron images recorded at energies of $-130.95 \mathrm{~cm}^{-1},-130.43 \mathrm{~cm}^{-1}$, and $-130.19 \mathrm{~cm}^{-1}$ corresponding to adjacent Stark states identified as $\left(n, n_{1}, n_{2}, m\right)=(31,7,23,0),(34,1,32,0)$ and $(27,26,0,0)$, respectively. (d)-(f) Comparison between the experimental radial distributions (solid line), and calculated radial distributions (dashed lines) obtained by means of TDSE calculations. Please note that, since $\mathrm{P}(\mathrm{R})=\int \mathrm{P}(\mathrm{R}, \alpha) \mathrm{Rd} \alpha$, the radial probability distributions $\mathrm{P}(\mathrm{R})$ have a zero at $\mathrm{R}=0$, even if the twodimensional images described in polar coordinates by $\mathrm{P}(\mathrm{R}, \alpha)$ do not. The main result is the appearance of a minimum in the radial distribution for the $(34,1,32,0)$ state, which corresponds to the value of $n_{1}$ and directly reveals the nodal structure of the $\chi_{1}(\xi)$ wave function. N.B. The computational results shown here and in Fig. 4 were scaled to the dimensions of the experiment in order that the best fit is achieved for the $(34,1,32,0)$ state. (g) Stark map for helium obtained from calculated photoabsorption cross sections (squares); superimposed dashed lines schematically indicate the field dependence of the related diabatic, uncoupled Stark states. The gray bar indicating the experimental field strength illustrates that the photoelectron image for the $(34,1,32,0)$ state was recorded near an avoided crossing of this red state with the blue $(27,26,0,0)$ state. (h) Field-dependent reduction in the linewidth of the $(34,1,32,0)$ state, extracted from the calculated photo-absorption spectra and confirming the occurrence of the interference narrowing effect.

case of the $(35,5,29,0)$ state shown in Fig. 4, the TDSE calculations revealed a three orders of magnitude drop in the ionization rate over a field range of $0.5 \mathrm{~V} / \mathrm{cm}$, ipso facto confirming the occurrence of the interference narrowing effect [Fig. 4(d)].

With this, the experiment can be fully explained. Given the Stark lifetime ( $\geq 100 \mathrm{ps})$ needed for the observation of
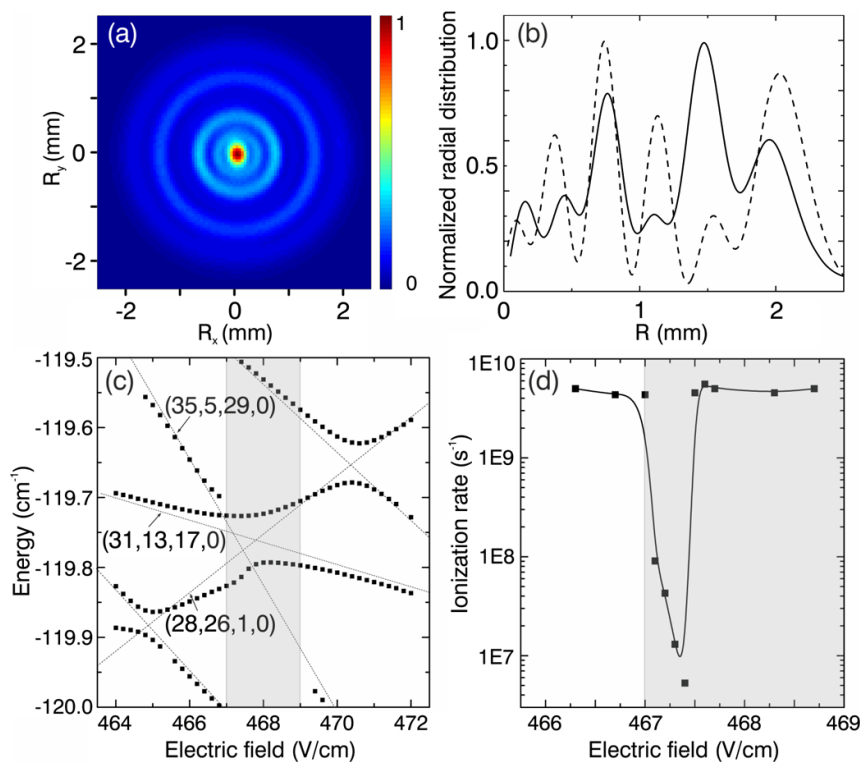

FIG. 4 (color online). (a) Raw photoelectron image for the $(35,5,29,0)$ state measured near an avoided crossing, showing five minima in the radial distribution, (b) comparison between the experimental (solid line) and theoretical radial distributions (dashed line, obtained from the TDSE calculations), showing an excellent agreement in the position of the nodes. A calculated Stark map (c) indicates that the measurement was taken for a dc electric field of approximately $467.4 \mathrm{~V} / \mathrm{cm}$, where the $(35,5,29$, $0)$ state has an avoided crossing with the $(31,13,17,0)$ and $(28$, $26,1,0)$ states. Note that the absence of black squares implies a disappearance of the oscillator strength in the photoabsorption spectra for a given state. The calculated ionization rate of the $(35,5,29,0)$ state, shown in (d), decreases by three orders of magnitude within a field range of $0.5 \mathrm{~V} / \mathrm{cm}$, due to the occurrence of interference narrowing.

sharply defined resonances in the photoexcitation efficiency as a function of laser wavelength, most measurements involved the excitation of quasibound Stark states in the middle or on the blue side of the Stark manifold. These states are strongly bound along both the $\xi$ and $\eta$ coordinates, and can only ionize via core-scattering into red continuum states where the $\eta$ motion is unbound. Consequently, as shown in Fig. 2, the interference patterns for these states no longer reveal a distinct value of $n_{1}$ and are fully determined by the phase differences that the photoelectrons accumulate along multiple classical paths that they can follow between the ion and the detector. In addition, we have performed a number of experiments where quasibound Rydberg states were excited on the red side of a Stark manifold, and in the vicinity of an avoided crossing with a blue state that induced an enhancement of the Rydberg lifetime via interference narrowing (Figs. 3 and 4). All these red states furthermore occurred at energies just below the parabolic critical energy, which marks the top of the $V(\eta)$ potential. Under these circumstances, the electron may leave the atom by a tunneling process that 
leaves the quantization along the $\xi$-coordinate intact. This ionization mechanism occurs in competition with the coreinduced coupling mechanism and is more prominent for atoms-like helium - with very small quantum defects $(\mu \ll 1)$ [31]. The interference narrowing is essential, because it causes a destructive interference between the bound-continuum coupling amplitudes of the crossing red and blue states, decoupling the red state from the ionization continuum. This causes hydrogenic tunneling to be the dominant ionization mechanism, thereby allowing the observation of the $n_{1}$ quantum number of the red Stark state on the detector [13]. Hence, the photoionization microscopy technique allows us to visualize the dc electric field-induced coupling between the red and blue Stark states in the vicinity of the avoided crossing. In the future, it will be of great interest to perform field-dependent experiments, mapping out the couplings that occur in Stark manifolds around field-induced avoided crossings. Furthermore, it will be of interest to investigate the influence on the Stark wave functions of other external perturbations (such as magnetic fields), or coupling of the electronic motion to internal (vibrational, rotational) degrees of freedom that exist in molecules.

The authors thank Dr. Wim Vassen for his advice on designing and building the metastable helium source. We acknowledge valuable contributions by Rob Kemper, Henk-Jan Boluijt, Iliya Cerjak, and Marco Konijnenburg. This work is part of the research program of the "Stichting voor Fundamenteel Onderzoek der Materie (FOM)," which is financially supported by the "Nederlandse organisatie voor Wetenschappelijk Onderzoek (NWO)." Francis Robicheaux is supported by the US DOE (Department of Energy). Tom Bergeman is supported by the US NSF.

*Corresponding author. marc.vrakking@mbi-berlin.de

[1] T. F. Gallagher, Rydberg Atoms (Cambridge University Press, Cambridge, England, 1994).

[2] R. R. Jones and L. D. Noordam, Adv. At. Mol. Opt. Phys. 38, 1 (1997).

[3] G. M. Lankhuijzen and L. D. Noordam, Adv. At. Mol. Opt. Phys. 38, 121 (1997).

[4] F. Merkt, Annu. Rev. Phys. Chem. 48, 675 (1997).

[5] H. H. Fielding, Annu. Rev. Phys. Chem. 56, 91 (2005).
[6] M. J. Seaton, Rep. Prog. Phys. 46, 167 (1983).

[7] M. G. Littman, M. M. Kash, and D. Kleppner, Phys. Rev. Lett. 41, 103 (1978).

[8] M. L. Zimmerman, M. G. Littman, M. M. Kash, and D. Kleppner, Phys. Rev. A 20, 2251 (1979).

[9] S. Feneuille, S. Liberman, E. Luckoenig, J. Pinard, and A. Taleb, J. Phys. B 15, 1205 (1982).

[10] J. Y. Liu, P. McNicholl, D. A. Harmin, J. Ivri, T. Bergeman, and H. J. Metcalf, Phys. Rev. Lett. 55, 189 (1985).

[11] P. McNicholl, T. Bergeman, and H. J. Metcalf, Phys. Rev. A 37, 3302 (1988).

[12] C. Nicole, H. L. Offerhaus, M. J. J. Vrakking, F. Lepine, and C. Bordas, Phys. Rev. Lett. 88, 133001 (2002).

[13] A. S. Stodolna, A. Rouzée, F. Lépine, S. Cohen, F. Robicheaux, A. Gijsbertsen, J. H. Jungmann, C. Bordas, and M. J. J. Vrakking, Phys. Rev. Lett. 110, 213001 (2013).

[14] S. Cohen, M. M. Harb, A. Ollagnier, F. Robicheaux, M. J. J. Vrakking, T. Barillot, F. Lépine, and C. Bordas, Phys. Rev. Lett. 110, 183001 (2013).

[15] Y. N. Demkov, V. D. Kondratovich, and V. N. Ostrovskii, JETP Lett. 34, 403 (1981).

[16] V. D. Kondratovich and V. N. Ostrovsky, J. Phys. B 17, 2011 (1984).

[17] V. D. Kondratovich and V. N. Ostrovsky, J. Phys. B 23, 3785 (1990).

[18] C. Blondel, C. Delsart, and F. Dulieu, Phys. Rev. Lett. 77, 3755 (1996).

[19] C. Bordas, F. Lepine, C. Nicole, and M. J. J. Vrakking, Phys. Rev. A 68, 012709 (2003).

[20] C. T. L. Smeenk, Physics 6, 58 (2013).

[21] L. D. Landau and E. M. Lifshitz, Quantum Mechanics: Non-relativistic Theory (Pergamon, New York, 1965).

[22] W. C. Martin, Phys. Rev. A 36, 3575 (1987).

[23] W. Rooijakkers, W. Hogervorst, and W. Vassen, Opt. Commun. 123, 321 (1996).

[24] A. T. J. B. Eppink and D. H. Parker, Rev. Sci. Instrum. 68, 3477 (1997).

[25] H. L. Offerhaus, C. Nicole, F. Lepine, C. Bordas, F. Rosca-Pruna, and M. J. J. Vrakking, Rev. Sci. Instrum. 72, 3245 (2001).

[26] L. B. Zhao and J. B. Delos, Phys. Rev. A 81, 053417 (2010).

[27] L. B. Zhao and J. B. Delos, Phys. Rev. A 81, 053418 (2010).

[28] F. Robicheaux and J. Shaw, Phys. Rev. Lett. 77, 4154 (1996).

[29] F. Robicheaux, Phys. Rev. A 56, 4032 (1997).

[30] H. J. Silverstone, Phys. Rev. A 18, 1853 (1978).

[31] D. H. Yang, D. Lieberman, P. VanderStraten, T. Bergeman, and H. Metcalf, Phys. Rev. A 40, 5026 (1989). 